\title{
Waldausdehnung in zwei Regionen des Schweizer Berggebiets: eine integrative Analyse
}

Robert Huber

Peter Bebi

Simon Briner

Harald Bugmann

Alexandre Buttler

Adrienne Grêt-Regamey

Christian Hirschi

Willi Zimmermann

Andreas Rigling

Eidgenössische Forschungsanstalt für Wald, Schnee und Landschaft $(\mathrm{CH})^{*}$

WSL-Institut für Schnee- und Lawinenforschung $(\mathrm{CH})$

Agri-food and Agri-environmental Economics Group, Institut für Umweltentscheidungen, ETH Zürich (CH)

Waldökologie, Institut für Terrestrische Ökosysteme, ETH Zürich (CH)

Laboratoire des systèmes écologiques, EPF Lausanne, und Eidgenössische Forschungsanstalt WSL (CH)

PLUS, Institut für Raum- und Landschaftsentwicklung, ETH Zürich $(\mathrm{CH})$

Umweltpolitik und Umweltökonomie, Institut für Umweltentscheidungen, ETH Zürich (CH)

Umweltpolitik und Umweltökonomie, Institut für Umweltentscheidungen, ETH Zürich (CH)

Eidgenössische Forschungsanstalt für Wald, Schnee und Landschaft $(\mathrm{CH})$

\section{Forest expansion in two Swiss mountain regions: an integrative analysis}

\begin{abstract}
The "Mountland" project analyzes climatic and land-use changes in mountain regions from an integrative perspective. One key interaction between the forest and agricultural sector is agricultural land abandonment and the subsequent forest expansion in mountain regions. To understand and assess this phenomenon, socioeconomic developments as well as ecological mechanisms of forest expansion must be taken into account. This article summarizes results from a simulation study of land abandonment applying the land-use model «Aluam» in two case study regions of Switzerland (Jura [VD] and Visp [VS]) which explicitly takes into account socioeconomic and ecological drivers. Two policy scenarios ("status quo" and "liberalization") including climate change projections are presented and discussed. Results show that land abandonment and the corresponding consequences for ecosystem services provision strongly differ between scenarios and case study regions. In addition to this heterogeneity, thresholds and interactions between economic and ecological performances are important aspects in the context of land abandonment. As a consequence, policy measures that should steer against land abandonment and subsequent forest expansion have to consider local conditions. Spatial planning methods and instruments represent key elements in this process.
\end{abstract}

Keywords: climate change, land-use change, land abandonment, forest expansion, ecosystem services, interdisciplinary research doi: $10.3188 /$ szf.2012.0502

* Zürcherstrasse 111, CH-8903 Birmensdorf, E-Mail robert.huber@wsl.ch

$\mathrm{D}$ ie land- und forstwirtschaftlichen Ökosysteme erbringen eine Vielzahl wichtiger Leistungen für die Bevölkerung (Millennium Ecosystem Assessment 2005). Sie bilden unter anderem die Grundlage für die Primärproduktion, d.h. die Herstellung von Nahrungsmitteln und Holz, bieten Schutz vor Naturgefahren und leisten einen wichtigen Beitrag zur Speicherung von Kohlenstoff (Olschewski \& Elsasser 2010). Gleichzeitig ist eine möglichst hohe Biodiversität eine Voraussetzung für die Belastbarkeit der Ökosysteme. Zukünftige Klimaund Landnutzungsänderungen werden das Funktionieren der Ökosysteme verändern und dadurch die Bereitstellung dieser Ökosystemleistungen beeinflussen (Kirchner 2008).

In den Berggebieten bewegt sich die Landwirtschaft im Spannungsfeld von wirtschaftlichem Druck und traditioneller Landnutzung. Fruchtbare und gut erschlossene Flächen werden intensiviert, mit oft negativen Folgen für die Umwelt (Stöcklin et al 2007), während Grenzstandorte verbrachen (Gellrich et al 2007). Gleichzeitig ist das wirtschaftliche Auskommen der Landwirte von staatlichen Subventionen abhängig. Auch die Zukunft der Waldwirtschaft ist, trotz einem möglichen Anstieg der Holzpreise, durch die geringe Rentabilität infrage gestellt (Gotsch et al 2004). Die lange Zeit auf die Primärproduktion ausgerichtete Land- und Forstwirtschaft wird zunehmend mit der Nachfrage nach öffentlichen Gütern und Dienstleistungen konfrontiert (Grêt-Regamey et al 2012). Der Anspruch unterschiedlicher gesellschaftlicher Akteure an den Schutz und die Bewirtschaftung von (Gebirgs-)Landschaften wird daher in privaten und öffentlichen Entscheidungsprozessen wichtiger werden (Lehmann \& Messerli 2007). 
In diesem Kontext leistet das inter- und transdisziplinäre Forschungsprojekt «Mountland» einen Beitrag zur Entwicklung eines nachhaltigen Landnutzungsmanagements und von Politikmassnahmen in Bergregionen (Huber et al 2012b). Der vorliegende Artikel illustriert Schlüsselergebnisse des Projekts mit Blick auf die Interaktionen zwischen Land- und Forstwirtschaft (Abbildung 1). Das klassische Beispiel dafür ist die Waldausdehnung im Berggebiet. Diese kann nur verstanden werden, wenn es gelingt, die Erkenntnisse in der sozioökonomischen Entwicklung der Landwirtschaft mit den naturwissenschaftlichen Kenntnissen der Waldentwicklung zu kombinieren (Bebi \& Baur 2002). Im Forschungsprojekt «Primalp» (Gotsch et al 2004) und im Nationalen Forschungsprogramm 48 «Landschaften und Lebensräume der Alpen» (Lehmann \& Messerli 2007) wurden die Ursachen der Waldausdehnung bereits untersucht. Der zusätzliche Beitrag von «Mountland» liegt darin, dass in diesem Projekt die identifizierten Ursachen in einen Modellverbund, bestehend aus ökologischen Prozessmodellen und einem ökonomischen Landnutzungsmodell, integriert und mit Klimaszenarien verbunden werden können (Abbildung 2).

Das Ziel ist, die enge Verbindung der beiden Sektoren in der Beurteilung von Ökosystemleistungen auf regionaler Landschaftsebene aufzuzeigen und die Auswirkungen dieser gemeinsamen Perspektive auf die Entwicklung von Politikmassnahmen zu analysieren. Aktualität erhält diese Forschung insbesondere durch die Anpassung des Waldgesetzes, welche eine Lockerung des Rodungsersatzes und die Möglichkeit der Einführung einer statischen Waldgrenze auch ausserhalb der Bauzone beinhaltet (Zimmermann 2012), sowie durch die anstehende Agrarreformetappe AP14-17 und die Revision des Raumplanungsgesetzes.

\section{Waldausdehnung in der Schweiz: Entwicklung, Ursachen und Aus- wirkungen auf Ökosystemleistungen}

Die Waldausdehnung im Berggebiet wird durch die Daten des Schweizerischen Landesforstinventars (LFI) und deren retrospektive Analyse illustriert (Ginzler et al 2011). Gemäss den neuesten Erhebungen nimmt die Waldfläche schweizweit jährlich um $0.5 \%$ zu. Es sind jedoch regional deutliche Unterschiede vorhanden. Zwischen dem ersten und dem dritten LFI (1983/1985-2004/2006) nahm die Waldfläche auf der Alpensüdseite um 16\% und in den Alpen um 15\% zu. Im Vergleich dazu war die Zunahme in den Voralpen (5\%) und im Jura (2.5\%) deutlich geringer, und im Mittelland (0.7\%) blieb die Waldfläche praktisch gleich (Brändli 2010). Diese Entwicklung wird auch durch die aktuellsten Zahlen des vierten LFI bestätigt. ${ }^{1}$ 1 Medienmitteilung von Bafu und WSL vom 21. März 2012
www.wsl.ch/medien/news/landesforstinventar_4_2012/index_
DE (4.10.2012).
Abb 1 Wytweiden wie hier im Waadtländer Jura sind ein klassisches Beispiel für die enge Verbindung von Landund Forstwirtschaft.

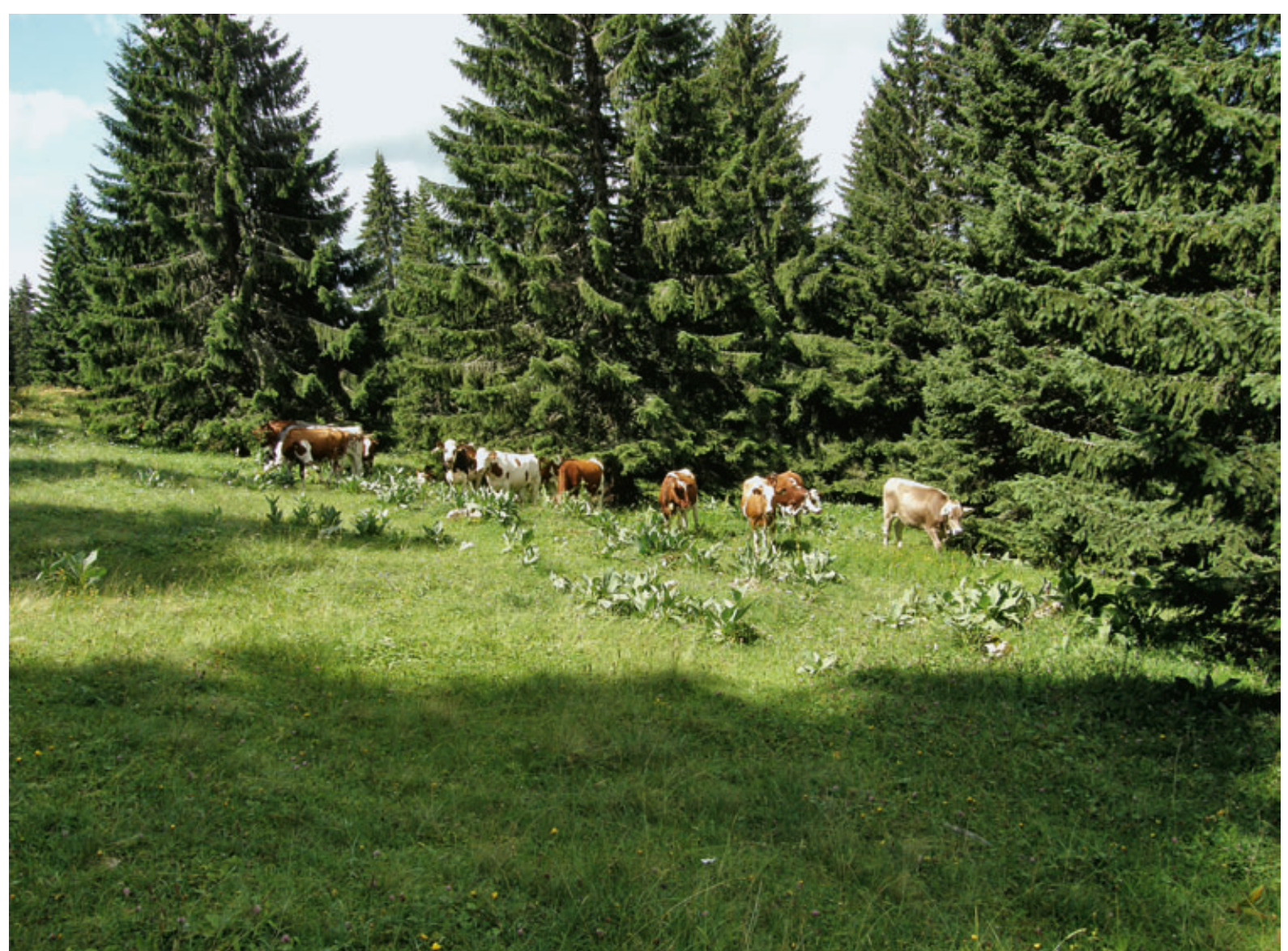




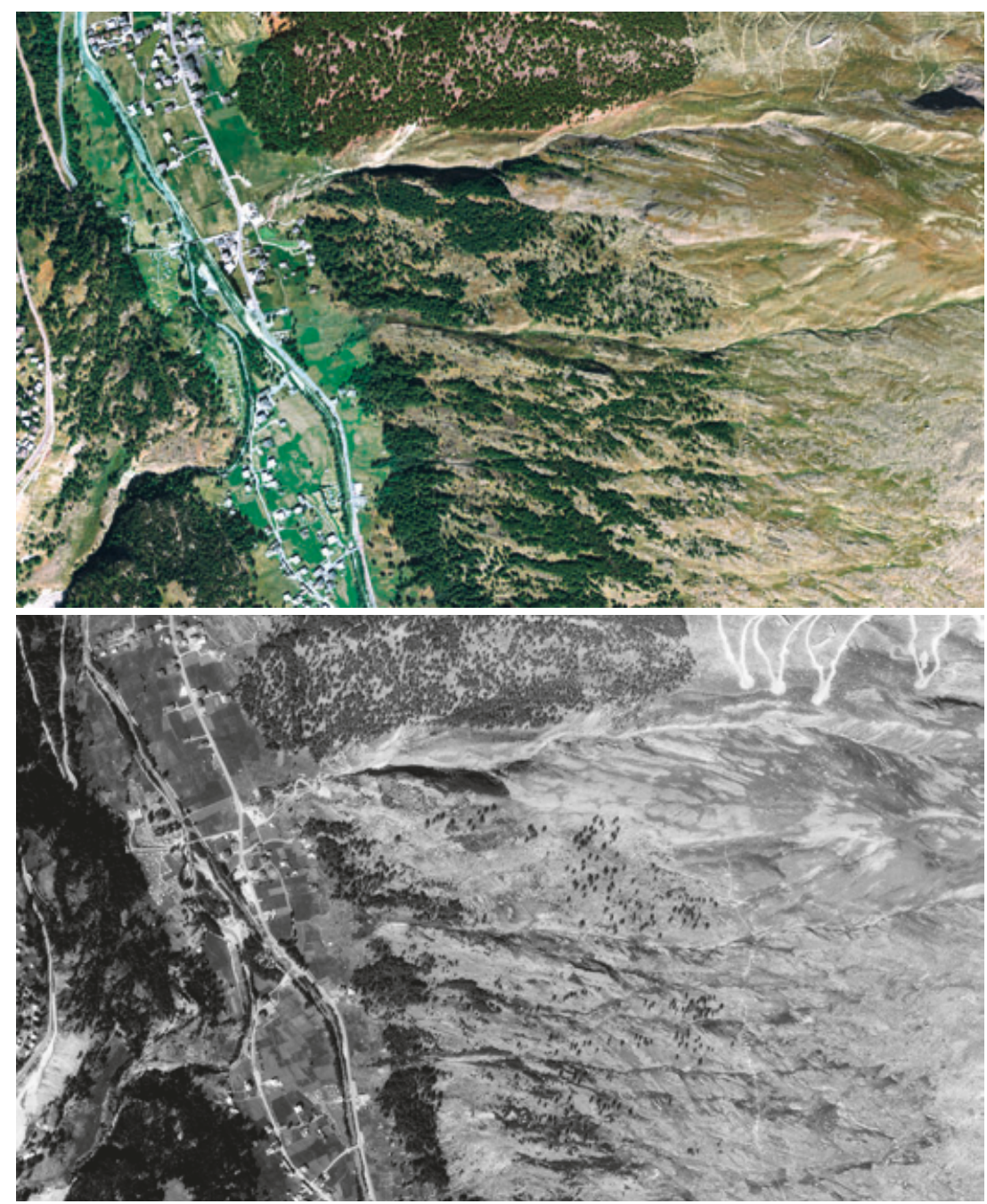

Abb 2 Waldausdehnung in der Fallstudienregion Visp zwischen 1980 (unten) und 2005 (oben). Luftbild reproduziert mit Bewilligung von swisstopo (JA100118), Orthofoto von Christian Ginzler, WSL
Die Ursachen und die Geschwindigkeit der Waldausdehnung sind komplex und hängen von verschiedenen Interaktionen ab (Bebi \& Baur 2002). Dazu gehören Standortfaktoren wie die Bodeneigenschaften, die Hangneigung oder die klimatischen Bedingungen, die alle auch einen Einfluss auf die Produktivität und die Kosten der Bewirtschaftung haben (Gellrich et al 2007). Die Waldflächenzunahme ist grösser und verläuft schneller, wenn auch benachbarte Gebiete am Einwachsen sind (Gellrich \& Zimmermann 2007). Agronomisch schlechte Standorte in höheren Lagen, die zudem oft schlecht erschlossen sind, werden eher aufgegeben und damit vom Wald zurückerobert (Baur 2006). Dabei spielt die Rationalisierung der landwirtschaftlichen Produktion eine wichtige Rolle. Die Ursachen einer fehlenden Rationalisierung sind beispielsweise tiefe Opportunitätskosten der Arbeit, ein hoher Anteil der Nebenerwerbslandwirtschaft, die Motivation und Einstellung der Landwirte, hoher Investitionsbedarf in Bezug auf Gebäude und Erschliessung sowie die Ausgestaltung des Direktzahlungssystems, insbesondere in seiner Wirkung auf extensive Bewirtschaftungsweisen (Gellrich et al 2008). Aus einer überge- ordneten Perspektive werden diese Faktoren von der Bevölkerungsentwicklung, der allgemeinen Wirtschaftsentwicklung und der Ausrichtung des Wirtschaftsstandorts beeinflusst (Flury et al 2013). In touristisch geprägten Gebieten ist eine geringere Waldzunahme zu erwarten (Bolliger et al 2007) als in wirtschaftlichen Randregionen. Im Weiteren spielen auch die historische Nutzung des Standorts und gesetzliche Regelungen wie beispielsweise die (frühere) Realteilung eine wichtige Rolle (Baur 2006).

Die Auswirkungen der Waldausdehnung auf die Ökosystemleistungen sind komplex (Keenleyside \& Tucker 2010). Grundsätzlich lassen sich jedoch folgende Wirkungen beschreiben:

1) Die Landwirtschaft verliert einen Teil ihrer Produktionsgrundlagen (auch wenn es sich vorwiegend um Grenzstandorte handelt).

2) Die Biodiversität nimmt tendenziell ab, da vielfach ökologisch wertvolle Biotope betroffen sind (Stöcklin et al 2007).

3) Die Holzressourcen und die $\mathrm{CO}_{2}$-Speicherung nehmen zu (Bolliger et al 2008).

4) Es ist davon auszugehen, dass mit der Ausdehnung des Waldes auch dessen Schutzleistungen zunehmen (Bebi et al 2012, Rigling et al 2012, beide dieses Heft).

5) Das Landschaftsbild verändert sich negativ, weil vollständig bewaldete Flächen im Berggebiet als weniger attraktiv eingeschätzt werden (Soliva et al 2010).

Die Folgen einer Waldausdehnung können nicht a priori als positiv oder negativ beurteilt werden. Während viele Studien zur Waldausdehnung in den Alpen ein eher negatives Bild zeichnen (Marini et al 2011, Flury et al 2013), gehen beispielsweise Renwick et al (2013) auf europäischer Ebene von einer positiven Umweltwirkung durch die Waldausdehnung aus.

\section{Modellbasierte Analyse der Wald- zunahme in den Fallbeispielregionen Jura und Visp}

In den Modellrechnungen wurde das Landnutzungsmodell «Aluam» verwendet, welches Landnutzungsänderungen im Berggebiet unter Berücksichtigung von klimatischen und sozioökonomischen Veränderungen simuliert (Briner et al 2012). Durch die Verbindung mit dem Landschaftsmodell «LandClim» (Elkin et al 2012, Schumacher et al 2004) in der Fallbeispielregion Visp und dem Vegetationsmodell «WoodPam» (Gillet 2008) im Jura können ökologische Waldentwicklungen im Kontext der Waldausdehnung räumlich explizit dargestellt werden. Die Erweiterung des Modells mit Agenten, welche einzelne Landwirtschaftsbetriebe oder Gruppen von Landwirten abbilden, ermöglicht es, auch die sozio- 


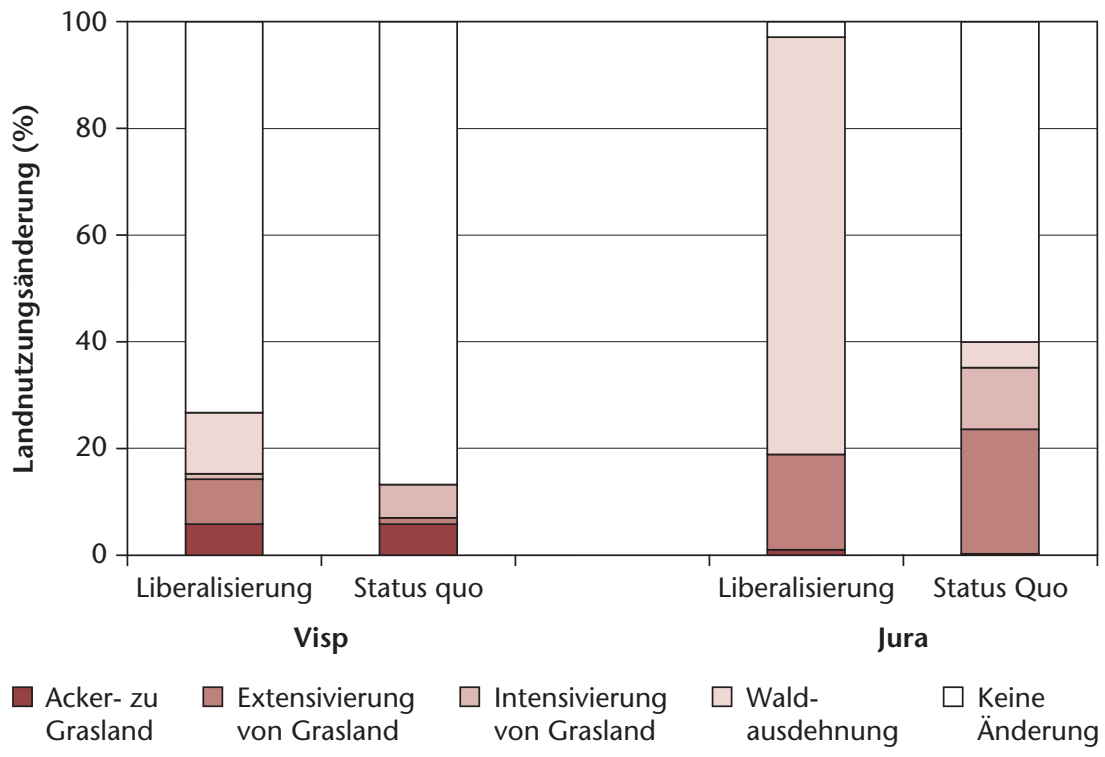

Abb 3 Landnutzungsänderung in den Fallbeispielregionen Visp und Jura bis 2030 in den Politikszenarien «Status quo» und «Liberalisierung».

ökonomischen Bedingungen der Waldausdehnung (Opportunitätskosten, Nebenerwerbslandwirtschaft, Direktzahlungssystem, Einstellung der Landwirte) zu berücksichtigen (Huber et al 2012a).

Im Rahmen von «Mountland» wurden Landnutzungsänderungen aufgrund von verschiedenen Klima- und Politikszenarien simuliert. In vorliegendem Artikel vorgestellt werden die Ergebnisse aus einem Klima- und zwei Politikszenarien. Es handelt sich dabei um das Klimaszenario A1, bei welchem mit einer Erhöhung der globalen Durchschnittstemperatur bis $2080 \mathrm{um} 4{ }^{\circ} \mathrm{C}$ gerechnet wird, sowie die Politikszenarien «Status quo» und «Liberalisierung». Beim Szenario «Status quo» bleiben das heutige Preisniveau für Agrarprodukte sowie das heutige Direktzahlungssystem bestehen. Beim Szenario «Liberalisierung» wird das EU-Preisniveau für Agrarprodukte angenommen, und das Direktzahlungssystem wird gemäss Botschaft des Bundesrats zur Agrarpolitik AP14-17 (BB1 2012 2075) geändert.
Abbildung 3 zeigt die simulierte Veränderung der Landnutzung in den Szenarien «Liberalisierung» und «Status quo» für die Fallstudiengebiete Visp und Jura im Jahr 2030. Die Waldausdehnung hängt dabei wesentlich vom betrachteten Politikszenario ab. Im Status-quo-Szenario ist sie in beiden Regionen gering und auf naturräumlich schlechte Standorte beschränkt. Im Szenario «Liberalisierung», in welchem die Landwirtschaft stark an Profitabilität verliert, nimmt die Waldausdehnung stärker respektive stark zu. Allerdings ist der Grad der Ausdehnung von den bestehenden landwirtschaftlichen Strukturen abhängig. Im Gegensatz zu den empirischen Analysen zeigt sich bei den Modellierungen, dass ein hoher Anteil an Nebenerwerbslandwirtschaft die Waldausdehnung nicht zwangsläufig begünstigt. Ausserlandwirtschaftliche Tätigkeiten ermöglichen in diesem Fall die Aufrechterhaltung einer (minimalen) Produktionsstruktur. Im Gegensatz dazu verschwindet im Liberalisierungsszenario die Hälfte der Vollerwerbsbetriebe im Jura, da diese finanziell stärker von der Produktion abhängig sind. Darüber hinaus wird die Waldausdehnung in den Modellierungen wesentlich von der Ausgestaltung der Direktzahlungen beeinflusst. So darf davon ausgegangen werden, dass mit der geplanten Umlagerung von tierbezogenen zu flächenbezogenen Direktzahlungen gemäss AP14-17 die Landwirtschaft viele Flächen weniger intensiv nutzen, jedoch nicht aufgeben würde (Huber et al 2012a).

Die Tabelle 1 zeigt die Entwicklung der landwirtschaftlichen Betriebe und von deren Einkommen sowie die aus den simulierten Landnutzungsänderungen abgeleiteten Auswirkungen auf die ausgewählten Ökosystemleistungen. Es lässt sich erkennen, dass in beiden Szenarien die meisten Indikatoren in die gleiche Richtung tendieren. Die Anzahl der Betriebe, das sektorale Einkommen und die Nahrungsmittelproduktion nehmen ab. Gleichzeitig verbessert sich die Bereitstellung jener Ökosys-

\begin{tabular}{|l|c|c|c|c|c|}
\hline \multirow{2}{*}{ Ökosystemleistung } & \multirow{2}{*}{$\begin{array}{c}\text { Jahr 2000 } \\
\text { \% }\end{array}$} & & \multicolumn{4}{|c|}{ Jahr 2030} \\
\cline { 3 - 6 } & & \multicolumn{2}{|c|}{ Visp } & \multicolumn{2}{|c|}{ Jura } \\
\cline { 3 - 6 } & & $\begin{array}{c}\text { Liberalisierung } \\
\%\end{array}$ & $\begin{array}{c}\text { Status quo } \\
\%\end{array}$ & $\begin{array}{c}\text { Liberalisierung } \\
\%\end{array}$ & $\begin{array}{c}\text { Status quo } \\
\%\end{array}$ \\
\hline Nahrungsmittelproduktion 1) & 100 & 70 & 90 & 27 & 92 \\
\hline CO $_{2}$-Ausstoss 2) & 100 & 68 & 74 & 19 & 92 \\
\hline Biodiversitätsindex Landwirtschaft 3) & 100 & 280 & 158 & 44 & 137 \\
\hline Schutzfunktion Wald 4) & 100 & 124 & 117 & - & 116 \\
\hline Landschaftsdiversitätsindex 5) & 100 & 102 & 104 & 49 & 85 \\
\hline Landwirtschaftliches Einkommen & 100 & 73 & 81 & 35 & 88 \\
\hline Anzahl Betriebe & 100 & 70 & 73 & 50 & - \\
\hline
\end{tabular}

Tab 1 Veränderung ausgewählter Ökosystemleistungen in den Fallbeispielregionen Visp und Jura bis 2030 in den Politikszenarien «Liberalisierung» und «Status quo». 1) Produzierte Nahrungsmittelmenge, ausgedrückt in Weizen-Äquivalenten (Briner et al 2012), 2) Treibhausgasausstoss in $t \mathrm{CO}_{2}$-Äquivalent, 3) Fläche der Trockenwiesen, die als extensiv genutzte Wiesen bewirtschaftet werden (Visp), respektive Fläche der extensiv genutzten Wiesen (Jura), 4) Schutzfunktion wurde nur für Visp beurteilt, berechnet wurde sie mithilfe eines Schutzindexes, der sowohl vom Zustand als auch vom Standort des Waldes abhängig ist, 5) Shannon-Diversitätsindex. 
temleistungen, welche mit einer Extensivierung der Landwirtschaft verbunden sind (Biodiversitätsindex, Landschaftsdiversitätsindex, $\mathrm{CO}_{2}$-Bilanz). Anders verhalten sich einzig der Biodiversitäts- und der Landschaftsdiversitätsindex im Jura unter dem Szenario «Liberalisierung»: Der Einkommensverlust ist dort so gross, dass die Anzahl der Betriebe um 50\% abnimmt und ein grosser Teil der Fläche nicht mehr bewirtschaftet wird (Abbildung 3). Dadurch homogenisiert sich die Landschaft, was zu einer Verschlechterung der Diversitätsindices führt. In der Region Visp führt die Zunahme der Waldfläche auch zu einem besseren Schutz vor Naturgefahren.

\section{Diskussion im Kontext von «Mountland»}

Diese Resultate widerspiegeln drei Aspekte, welche sich in der Synthese des Projekts «Mountland» als zentral für eine nachhaltige Landnutzung im Berggebiet herausstellten: Schwellenwerte, Heterogenität und Wechselbeziehungen.

\section{Schwellenwerte}

Ein Schwellenwert bezeichnet einen Punkt, an dem eine scheinbar linear oder zumindest monoton verlaufende Entwicklung sich ändert und in einen komplett neuen Zustand übergeht, der sich qualitativ von der ursprünglichen Situation unterscheidet (Groffman et al 2006). Im Jura zeigt sich ein solcher Schwellenwert in der Modellierung: Das gewählte Liberalisierungsszenario führt zu einem starken Rückgang der Einkommen; erreicht dieser Rückgang für die Vollerwerbsbetriebe im Jura ein gewisses Ausmass, so halbiert sich die Anzahl der Betriebe. Im Falle einer Status-quo-Politik bleibt hingegen die Anzahl der Betriebe auf einem wesentlich höheren Niveau. Im Gegensatz dazu kann in der Simulation für Visp kein solcher Schwellenwert beobachtet werden. Durch den bereits heute hohen Anteil an Nebenerwerbsbetrieben, bei denen die Profitabilität der Landwirtschaft nicht zentral ist, bleiben die Strukturen mittelfristig erhalten. Zudem handelt es sich bei den verbleibenden Vollerwerbsbetrieben um wachstumswillige, grosse Betriebe, die in neue Ställe investiert haben (Huber et al 2012a). Ein neuer Schwellenwert könnte sich durch die Änderung der ausserlandwirtschaftlichen Beschäftigungsmöglichkeiten ergeben. Ein Verlust von regionalen Arbeitsplätzen würde die Nebenerwerbslandwirtschaft in der Region Visp wesentlich erschweren. Die heute vergleichsweise stabilen Strukturen würden dadurch aufgebrochen, was der Waldausdehnung Vorschub leisten würde. Dieser Effekt könnte im Modell mithilfe von exogenen Annahmen berücksichtigt und mit Sensitivitätsanalysen überprüft werden, wie Lauber (2006) oder Flury (2002) zeigten. Zudem besteht Unsicherheit darüber, wie stabil die Präferenzen der Nebenerwerbslandwirte in der Region Visp sind. Zurzeit sind für die Bevölkerung lokale Traditionen ein wichtiger Aspekt der regionalen Entwicklung (Brand et al, accepted). Ob diese allerdings auch langfristig erhalten bleiben, wie in der Modellierung angenommen wird, ist offen (Brown \& Robinson 2006).

Neben diesen sozioökonomisch bedingten Schwellenwerten können sich auch die ökologischen Bedingungen abrupt ändern. So zeigen beispielsweise Rigling et al (2012, dieses Heft), dass die Biomasse in den Wäldern der Tieflagen der Fallbeispielregion Visp bis 2050 leicht abnimmt, die Baumartenzusammensetzung aber bestehen bleibt. Aufgrund einer Serie von Trockenjahren prognostiziert das Landschaftsmodell danach aber eine abrupte Änderung der Baumartenzusammensetzung.

\section{Heterogenität}

Die natürlichen Gegebenheiten und der sozioökonomische Kontext spielen eine zentrale Rolle für die Wirkung einer Politikmassnahme (Huber et al 2012a). So führt das gleiche Szenario in beiden Fallbeispielregionen zwar tendenziell zu ähnlichen Entwicklungen. Im Gegensatz zum Jura kann aber die Region Visp von einer Waldausdehnung direkt profitieren, da sich dadurch auch die Schutzleistungen der Wälder erhöhen. Daneben gibt es auch Unterschiede innerhalb der Regionen. Briner et al (2012) zeigen für die Region Visp, dass die Aufgabe der landwirtschaftlichen Nutzung abhängig von der Höhenstufe ist. Die Karten in Abbildung 4 illustrieren die räumlich heterogene Veränderung der Landnutzung in den Szenarien «Liberalisierung» und «Status quo» für die Fallstudiengebiete Visp bis ins Jahr 2030.

Dabei spielen die Erträge eine wichtige Rolle. Wenn in den oberen Lagen die Verfügbarkeit von Gras für die Fütterung der Tiere aufgrund einer verlängerten Vegetationsperiode zunimmt, dann nimmt die Flächennutzung auch bei einer konstanten Tierzahl ab, da auf der gleichen Fläche mehr Futter zur Verfügung steht. In der Folge würde sich der Wald auch ohne zusätzlichen ökonomischen Druck ausdehnen. In den mittleren Höhenlagen hingegen ist aufgrund der hohen flächenbezogenen Direktzahlungen mit einer Extensivierung, nicht jedoch mit einer Aufgabe von Flächen zu rechnen. Neben der agronomischen Perspektive spielt aber auch die räumliche Heterogenität der zukünftigen Waldausdehnung eine Rolle. Bebi et al (2012, dieses Heft) zeigen für Davos, dass die Waldausdehnung in erster Linie unterhalb der Baumgrenze und nur in geringerem Ausmass an der Baumgrenze selbst zu erwarten ist. Eine Erhöhung der Durchschnittstemperatur ist daher nicht automatisch mit einem Höhersteigen einer geschlossenen Waldgrenze verbunden (Bebi et al 2012, dieses Heft). In der Region Visp ist in den tieferen Lagen, im Gegensatz zu den mittleren und hö- 


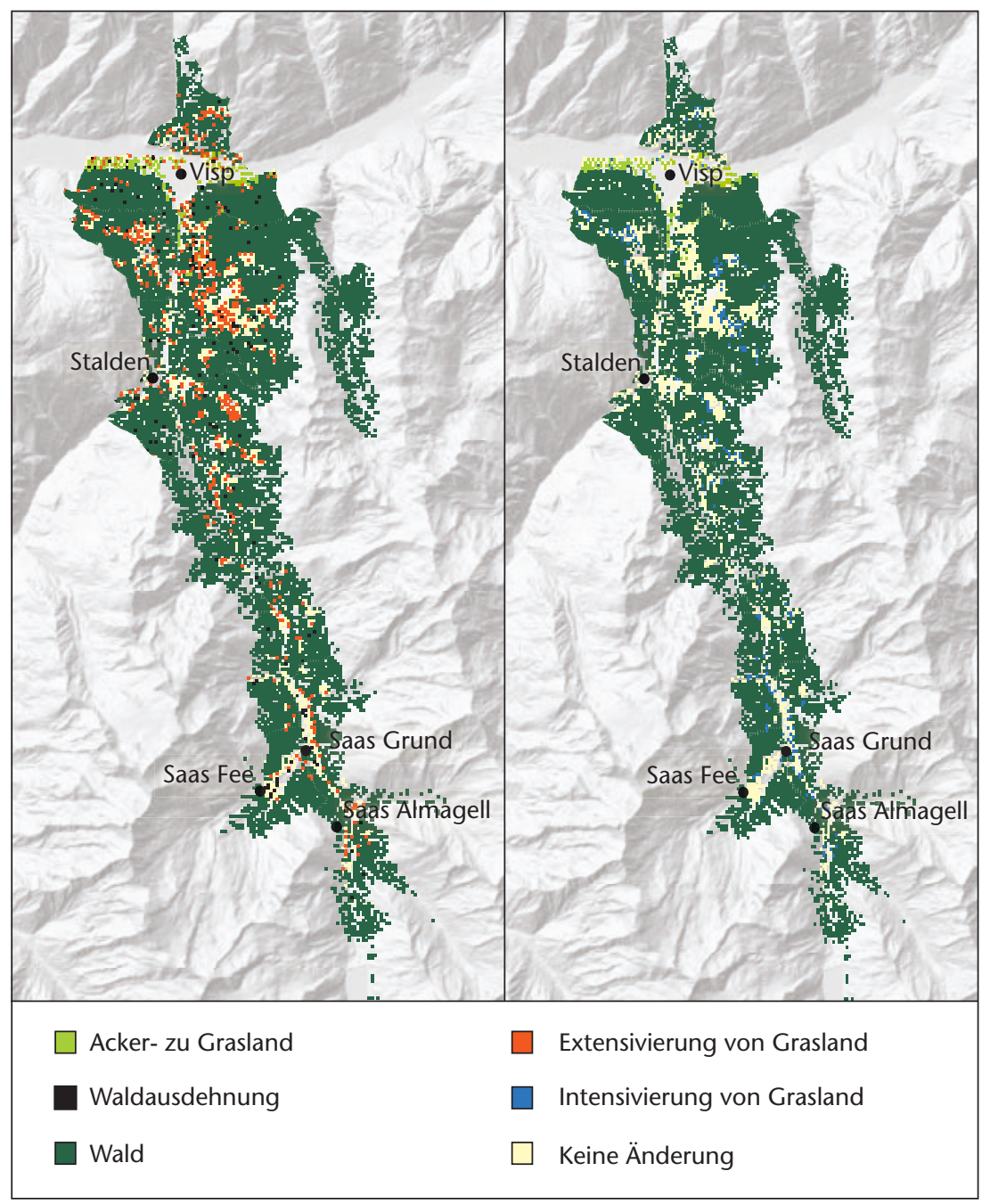

Abb 4 Simulierte Veränderung der landwirtschaftlichen Nutzung in der Fallbeispielregion Visp für die Szenarien «Liberalisierung» (links) und «Status quo» (rechts) bis zum Jahr 2030.

\section{Wechselbeziehungen}

Wechselbeziehungen sind in der multifunktionalen Land- und Forstwirtschaft allgegenwärtig. In den meisten Fällen nimmt mit einer Zunahme der Nutzung (bzw. der Produktion) die Bereitstellung anderer Ökosystemleistungen, die nicht monetär abgegolten werden, zuerst $\mathrm{zu}$, mit einer weiteren Intensivierung dann jedoch ab (Harvey 2003). Dadurch können Austauschbeziehungen oder Zielkonflikte (engl. Trade-offs) zwischen der Produktionsleistung und anderen Ökosystemleistungen entstehen. Die Modellrechnungen von «Mountland» offenbaren solche Zielkonflikte in beiden Fallbeispielregionen. So wird in allen Szenarien ein Rückgang der landwirtschaftlichen Nahrungsmittelproduktion simuliert (Tabelle 1). Dies führt zu einer Verbesserung der $\mathrm{CO}_{2}$-Bilanz, da die Emission von äusserst klimarelevanten Treibhausgasen aus der Landwirtschaft (Methan, Lachgas) stark zurückgeht. Zudem ermöglicht der Rückgang der Nahrungsmittelproduktion eine extensivere Nutzung der Flächen, wodurch der landwirtschaftliche Biodiversitätsindex ansteigt. Nimmt dann aber die Waldausdehnung zu stark zu, wie beispielsweise im Liberalisierungsszenario im Jura, nimmt der landwirtschaftliche Biodiversitätsindex wieder ab, weil ökologisch wertvolle Flächen an der Schnittstelle zwischen Wald- und Landwirtschaft verloren gehen. Auch in den anderen beiden Fallstudiengebieten führt die Ausdehnung des Waldes zu einer Homogenisierung der Landschaft (Briner et al 2012, Kulakowski et al 2011). Damit verbunden ist auch ein Anstieg der $\mathrm{CO}_{2}$-Speicherung und - sofern Gefahren- und Schadenpotenzial vorhanden sind eine Zunahme der Schutzfunktion des Waldes. Dabei ist zu beachten, dass die Schutzfunktion von der Waldentwicklung abhängt. In einem sehr frühen Sukzessionsstadium kann sich unter Umständen eine erhöhte Gefahr für Gleitschneelawinen und Hangrutschungen ergeben (Newesely et al 2000, Tasser et al 2003). Zudem kann auch die Veränderung der Baumartenzusammensetzung zu einer Reduktion der Schutzfunktion führen. Bebi et al (2012, dieses Heft) erwarten aufgrund ihrer Modellierungen (die sich auf den Wald und die Waldleistungen beschränken), dass nicht nur die Schutzfunktion und die $\mathrm{CO}_{2}$-Speicherung zunehmen, sondern auch der Erholungswert des Waldes.

Für die Einordnung der Resultate des Projekts «Mountland» sind zwei weitere Aspekte von Bedeutung: die sogenannten «Off-site»-Effekte und die Eintretenswahrscheinlichkeit der Modellszenarien.

Die gewählte regionale Perspektive erlaubt es nicht, jene Effekte abzubilden, welche ausserhalb der Region entstehen, jedoch in Wechselwirkung mit dem Geschehen in der Region stehen (sog. «Off-site»Effekte; Seppelt et al 2011). So bleiben beispielsweise Veränderungen in der Nahrungsmittelproduktion ausserhalb der Fallbeispielregion im Modell unbe- 


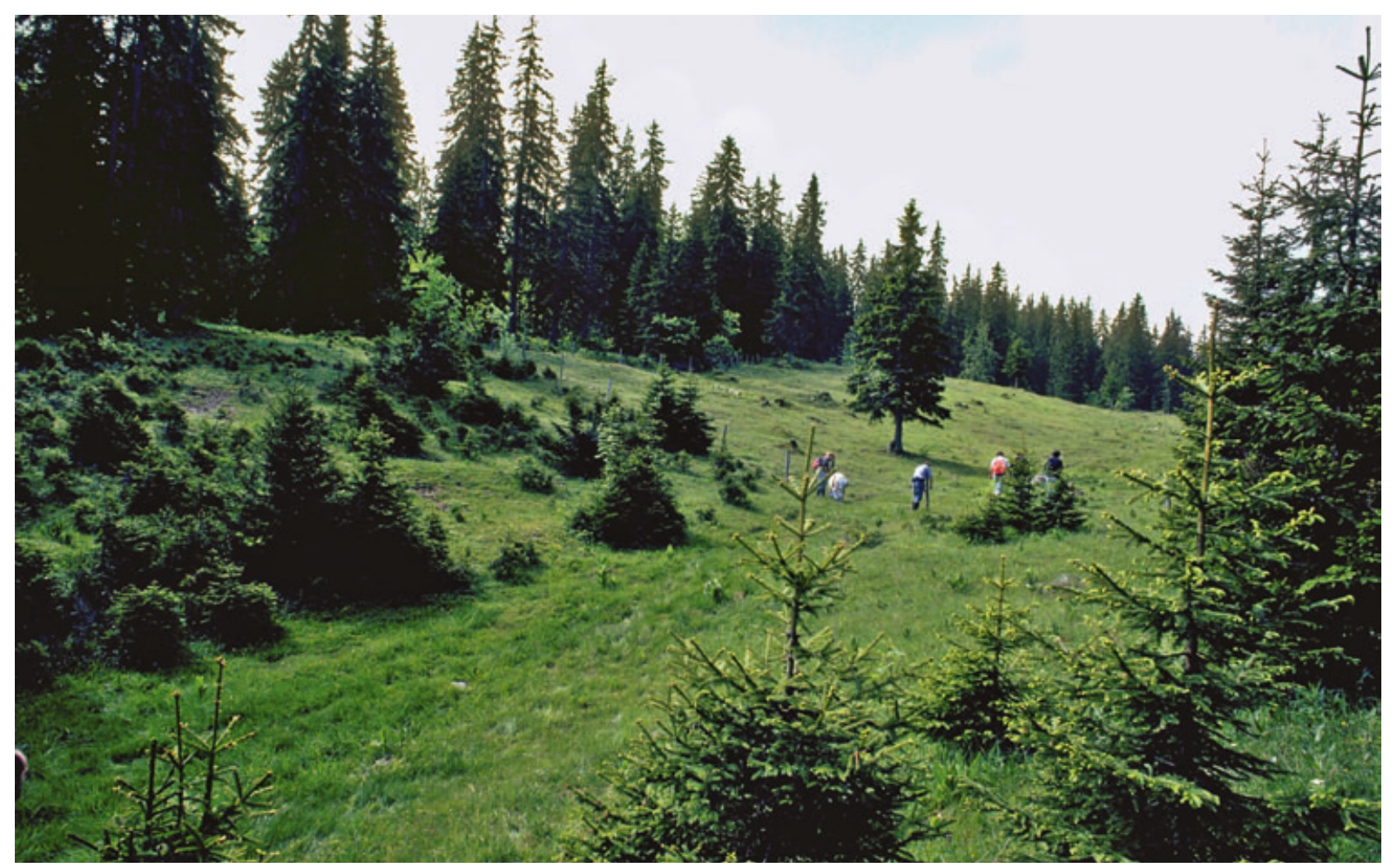

Abb 5 Waldausdehnung in den Wytweiden der Fallstudienregion Jura. Foto: Projekt Patubois

rücksichtigt. Auch werden die Kosten, die dem Steuerzahler beziehungsweise dem Konsumenten durch den Grenzschutz (Status-quo-Szenario) und die Direktzahlungen (beide Szenarien) entstehen, vernachlässigt. Die Resultate können damit nicht direkt zur Bewertung der beiden Politikszenarien verwendet werden. Dazu wäre es notwendig, neben dem Angebot an Ökosystemleistungen und privaten Gütern auch die Nachfrage nach denselben räumlich explizit zu erfassen und zu quantifizieren. Erst dann können die Auswirkungen von Politikoptionen auf die Ökosystemleistungen tatsächlich bewertet werden (Grêt-Regamey et al 2012).

Die bestehenden politischen Institutionen und deren Entwicklung haben einen grossen Einfluss auf die Eintretenswahrscheinlichkeit der Modellresultate. Vor dem Hintergrund des bestehenden Netzwerks in der schweizerischen Agrarpolitik ist das verwendete Szenario «Liberalisierung», dem das EU-Preisniveau für Agrarprodukte zugrunde liegt, mittelfristig unwahrscheinlich (Hirschi \& Huber 2012), und die Waldpolitik hat nur geringen Einfluss auf die Waldausdehnung im Schweizer Berggebiet (Hirschi et al 2012, dieses Heft).

Schliesslich müssen die Resultate auch mit Blick auf die methodischen Einschränkungen des Modells eingeordnet werden. Landnutzungsmodelle wie «Aluam» eignen sich gut für die Simulation von Zukunftsszenarien. Sie können aber nicht die Realität in all ihren Facetten wiedergeben und exakte Vorhersagen für die Zukunft machen. Die Resultate müssen daher immer mit Blick auf die getroffenen Annahmen interpretiert werden.

\section{Konsequenzen für die Politik- formulierung}

Mit Blick auf die diskutierten Aspekte Schwellenwerte, Heterogenität und Wechselbeziehungen lassen sich drei Folgerungen für die Politik mit Bezug zur Offenhaltung im Berggebiet formulieren:

\section{Schwellenwerte}

Sofern die Waldzunahme vermindert werden soll, ist es zentral, dass in der politischen Planung flexiblen Anpassungsmassnahmen Vorrang gegeben wird. Quantitative Vorgaben für die landwirtschaftlichen oder raumplanerischen Nutzungsplanungen, wie beispielsweise ein starrer Schutz von Landwirtschafts- oder Waldflächen, können durch abrupte sozioökonomische oder ökologische Entwicklungen unterlaufen werden. Wichtig dabei ist die Koordination der verschiedenen Politiken (Raumplanung, Agrarpolitik und Waldpolitik; Hirschi et al 2012, dieses Heft). Dadurch kann sichergestellt werden, dass ein Set von Politikoptionen zur Verfügung steht, welches auch bei unvorhergesehenen und abrupten Änderungen Handlungsoptionen bietet.

\section{Heterogenität}

Die Folge der grossen räumlichen Heterogenität ist, dass Massnahmen zur Verhinderung der Waldausdehnung räumlich explizit und damit den lokalen Verhältnissen angepasst sein müssen, wenn sie Erfolg haben sollen. Diese Erkenntnis ist nicht neu, eine entsprechende Politikempfehlung wurde bereits in «Primalp» (Flury et al 2004) und im NFP 48 


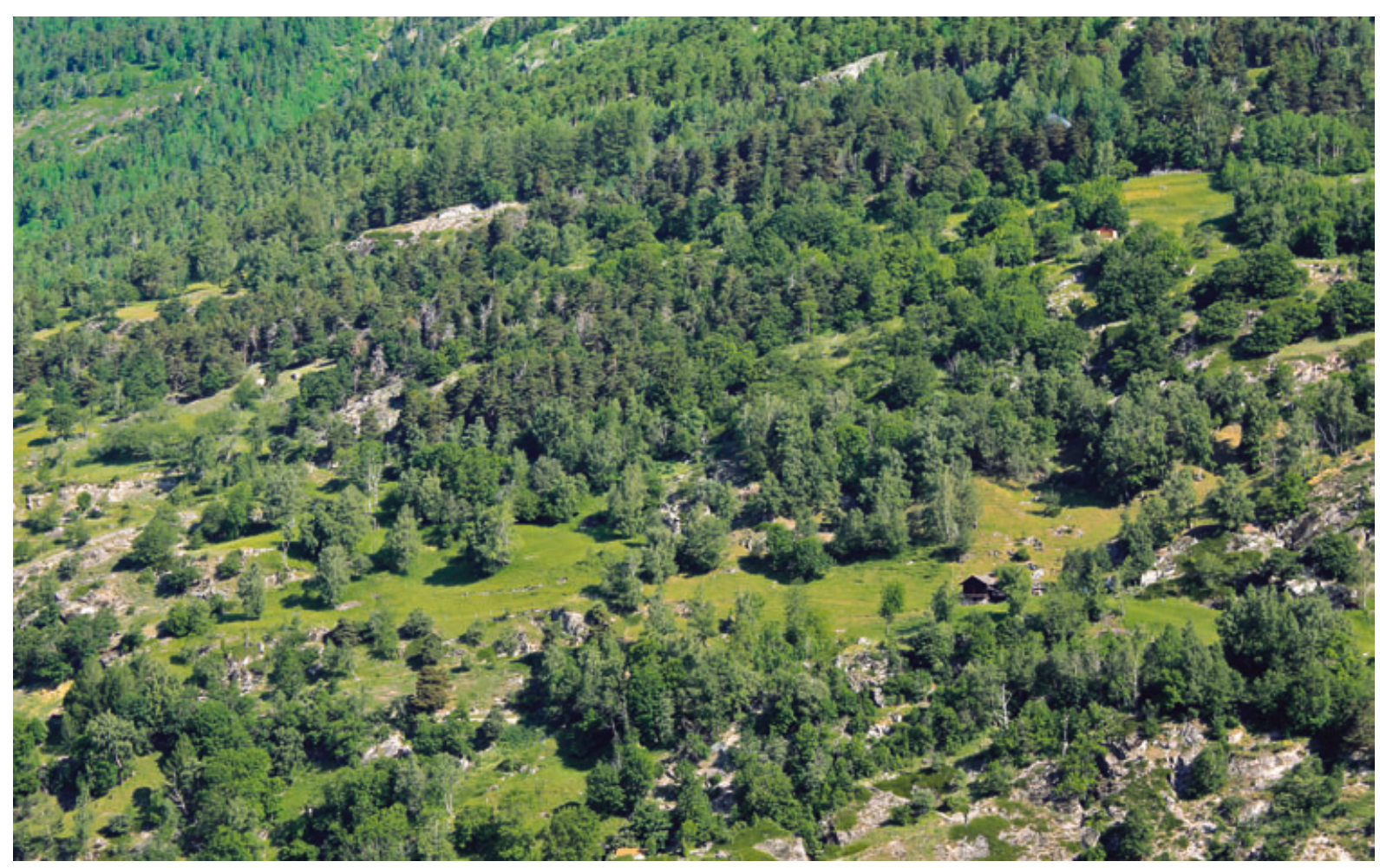

Abb 6 Einwachsende Landwirtschaftsflächen oberhalb Baltschieder in der Fallstudienregion Visp.

(Baur 2006, Gellrich et al 2008) formuliert. Die Resultate von «Mountland» zeigen aber, dass der Klimawandel die Notwendigkeit von föderalistischen Lösungen zur Eindämmung der Waldausdehnung noch verstärkt, indem 1) auf Grenzstandorten die klimabedingte Ausdehnung des Waldes die Nutzung des agronomischen Potenzials zusätzlich limitieren kann und 2) gut nutzbare landwirtschaftliche Flächen intensiver genutzt werden können (Jahresproduktion), wodurch schlechtere Flächen nicht mehr zur Deckung des Futterbedarfs benötigt werden (Abbildung 6). Beide Effekte führen zu einer verstärkten Waldausdehnung.

\section{Wechselbeziehungen}

Wechselbeziehungen und damit in erster Linie Zielkonflikte in der Erbringung von Ökosystemleistungen sollten in der Formulierung zukünftiger politischer Massnahmen zur Offenhaltung explizit berücksichtigt werden. Eine wichtige Folgerung des NFP 48 war, dass nicht alle Landschafts- beziehungsweise Ökosystemleistungen an jedem Ort bereitgestellt werden sollen, da dies ungewollt zu einer Monokultur führt (Lehmann \& Messerli 2007). Die «Mountland»-Resultate zeigen, dass die sorgfältige Berücksichtigung von räumlich expliziten Austauschbeziehungen zu Win-win-Situationen führen kann und die Fokussierung auf ausgewählte Ökosystemleistungen in verschiedenen Teilräumen einen effizienteren Einsatz öffentlicher Mittel erlauben würde. Instrumente und Methoden der Raumplanung bieten sich als Umsetzungsinstrumente an und können so zur Schaffung von Win-win-Situationen beitragen.

In konkreten Projekten zur Verhinderung (oder Duldung) der klimatisch und sozioökonomisch bedingten Waldausdehnung werden, neben der Landwirtschaftspolitik, raumplanerische Instrumente und Methoden zu politischen Schlüsselmechanismen, weil sie bestehende Wechselbeziehungen zwischen land- und forstwirtschaftlichen Ökosystemleistungen räumlich explizit berücksichtigen können. Darüber hinaus lassen sich auch flexible Massnahmen und projektorientierte Planungen mit ausreichender Berücksichtigung von lokalen Akteuren gut in die Raumplanung integrieren. Dies im Gegensatz zur Agrar- und Waldpolitik, welche als Bundespolitiken in dieser Hinsicht über weniger Spielraum verfügen. Projektbezogene Massnahmen (wie beispielsweise im Rahmen von Meliorationen) bieten die Möglichkeit einer sektorübergreifenden Umsetzung, die von den Betroffenen mitgestaltet werden kann.

Diese Folgerungen haben bereits eine erste Anwendung in dem vom Bund und dem Kanton Wallis entwickelten Leitfaden zum Vorgehen für Gemeinden mit Waldeinwuchs im Wallis gefunden (DWL \& Bafu 2011). Darin werden in Zusammenarbeit mit den lokalen Behörden und basierend auf den raumplanerischen Vorgaben zuerst Fokusräume und schliesslich sogenannte Schlüsselgelände definiert, auf welche die Umsetzung von Offenhaltungsmassnahmen fokussiert wird. Die spezifischen Massnahmen werden in einem zweiten Schritt definiert und mit lokalen Akteuren auf ihre Umsetzbarkeit 
hin geprüft. Grundlagen dazu kann auch ein neuer Bericht aus dem Forschungsprogramm «Agri Montana» bieten (Bollmann et al 2012), der Minimalnutzungsverfahren für die Offenhaltung der Kulturlandschaft definiert und beurteilt. Für die Praxis steht damit auch ein spezifisches Werkzeug zur Verfügung, das eine gute Grundlage darstellt.

Für die Umsetzung ist jedoch auch die Finanzierung der entsprechenden Massnahmen elementar. Zukünftige agrarpolitische Reformen sollten flexible Massnahmen zur Offenhaltung (z.B. Landschaftsqualitätsbeiträge) unterstützen, statt flächendeckende Zahlungen auszurichten. Dies würde es auf eine effiziente Weise ermöglichen, mithilfe von raumplanerischen Instrumenten die Waldausdehnung dort zu verhindern, wo aus ökosystemarer und gesellschaftlicher Sicht tatsächlich ein Bedarf besteht.

Eingereicht: 30. April 2012, akzeptiert (mit Review): 22. Oktober 2012

\section{Literatur}

BAUR P (2006) Die Rückkehr des Waldes im südlichen Alpenraum der Schweiz: Hintergründe eines Landschaftswandels. Schweiz Ges Agrarwirtschaft Agrarsoziologie 2/06: 3-26.

BEBI P ET AL (2012) Veränderung von Wald und Waldleistungen in der Landschaft Davos im Zuge des Klimawandels. Schweiz Z Forstwes 163: 493-501. doi: 10.3188/szf.2012.0493

BEBI P, BAUR P (2002) Forest expansion in the Swiss Alps: A quantitative analysis of bio-physical and socio-economic causes. Aust J For Sci 119: 217-230.

BOLLIGER J ET AL (2008) Effects of land-use change on carbon stocks in Switzerland. Ecosystems 11: 895-907.

BOLLIGER J, KIENAST F, SOLIVA R, RUTHERFORD G (2007) Spatial sensitivity of species habitat patterns to scenarios of land use change (Switzerland). Landsc Ecol 22: 773-789.

BOLLMANN R, SCHNEIDER M, FLURY C (2012) Minimalnutzungsverfahren zur Offenhaltung der Kulturlandschaft. ReckenholzTänikon: Agroscope. 41 p.

BRAND F, SEIDL R, LE QB, BRÄNDLE J, SCHOLZ RW (ACCEPTED) Constructing consistent multiscale scenarios by transdisciplinary processes: The case of mountain regions facing global change. Ecol Soc.

BRÄNDLI UB, EDITOR (2010) Schweizerisches Landesforstinventar. Ergebnisse der dritten Erhebung 2004-2006. Birmensdorf: Eidgenöss Forsch.anstalt WSL. 312 p.

BRINER S, HUBER R, ELKIN C, GRÊT-REGAMEY A (2012) Assessing the impacts of economic and climate changes on land-use in mountain regions: A spatial dynamic modeling approach. Agric Ecosyst Environ 149: 50-63.

BROWN DG, ROBINSON DT (2006) Effects of heterogeneity in residential preferences on an agent-based model of urban sprawl. Ecol Society 11: 46.

DWL, BAFU (2011) Leitfaden zum Vorgehen für Gemeinden mit Waldeinwuchs im Wallis. Sion: Dienststelle für Wald und Landschaft. $24 \mathrm{p}$.

ELKIN C, REINEKING B, BIGLER C, BUGMANN H (2012) Do smallgrain processes matter for landscape scale questions? Sensitivity of a forest landscape model to the formulation of tree growth rate. Landsc Ecol 5: 697-711.

FLURY C (2002) Zukunftsfähige Landwirtschaft im Alpenraum. Entwicklung von Nutzungsstrategien für den Kanton Graubünden auf der Basis eines Sektormodells. Kiel: Vauk. 268 p.
FLURY C, GOTSCH N, RIEDER P (2005) Site-specific and regionally optimal direct payments for mountain agriculture. Land Use Pol 22: 207-214.

FLURY C, HUBER R, TASSER E (2013) Future of mountain agriculture in the Alps. In: Mann S, editor. The future of mountain agriculture. Heidelberg: Springer.

GELLRICH M, BAUR P, KOCH B, ZIMMERMANN NE (2007) Agricultural land abandonment and natural forest re-growth in the Swiss mountains: A spatially explicit economic analysis. Agric Ecosyst Environ 118: 93-108.

GELLRICH M, BAUR P, ROBINSON BH, BEBI P (2008) Combining classification tree analyses with interviews to study why subalpine grasslands sometimes revert to forest: A case study from the Swiss Alps. Agric Syst 96: 124-138.

GELLRICH M, ZIMMERMANN NE (2007) Investigating the regional-scale pattern of agricultural land abandonment in the Swiss mountains: A spatial statistical modelling approach. Landsc Urb Plan 79: 65-76.

GILLET F (2008) Modelling vegetation dynamics in heterogeneous pasture-woodland landscapes. Ecol Model 217: 1-18.

GINZLER C, BRÄNDLI UB, HÄGELI M (2011) Waldflächenentwicklung der letzten 120 Jahre in der Schweiz. Schweiz Z Forstwes 162: 337-343. doi: 10.3188/szf.2011.0337

GOTSCH N (2004) Land- und Forstwirtschaft im Alpenraum Zukunft im Wandel. Synthesebericht des Polyprojektes «PRIMALP - Nachhaltige Primärproduktion am Beispiel des Alpenraums . Kiel: Vauk. 305 p.

GRÊT-REGAMEY A, BRUNNER SH, KIENAST F (2012) Mountain ecosystems Services: Who cares? Mt Res Dev 32 (S1): 23-34.

GROFFMAN P ET AL (2006) Ecological thresholds: The key to successful environmental management or an important concept with no practical application? Ecosystems 9: 1-13.

HARVEY DR (2003) Agri-environmental relationships and multifunctionality: Further considerations. The World Economy 26: 705-725.

HIRSCHI C, HUBER R (2012) Ökologisierung der Landwirtschaft im agrarpolitischen Prozess. Agrarforschung Schweiz 3: 360-365.

HIRSCHI C, WIDMER A, ZIMMERMANN W (2012) Waldausdehnung im Berggebiet: Prozesse und Entwicklungen in der Schweizer Waldpolitik. Schweiz Z Forstwes 163: 512-520. doi: 10.3188/szf.2012.0512

HUBER R, ITEN A, BRINER S (2012A) Weiterentwicklung des Direktzahlungssystems: Auswirkungen auf die Landnutzung im Berggebiet. Agrarforschung Schweiz 3: 354-359.

HUBER R, WALZ A, RIGLING A (2012B) Nachhaltige Land- und Forstwirtschaft im Berggebiet: Das Forschungsprojekt «Mountland». Schweiz Z Forstwes 163: 464-468. doi: 10.3188/szf.2012.0464

KEENLEYSIDE C, TUCKER GM (2010) Farmland abandonment in the EU: An assessment of trends and prospects. London: Institute European Environmental Policy. 96 p.

KIRCHNER JW (2008) Herausforderungen für Forstwirtschaft und Waldforschung im 21. Jahrhundert (Essay). Schweiz Z Forstwes 159: 224-227. doi: 10.3188/szf.2008.0224

KULAKOWSKI D, BEBI P, RIXEN C (2011) The interacting effects of land use change, climate change and suppression of natural disturbances on landscape forest structure in the Swiss Alps. Oikos 120: 216-225.

LAUBER S (2006) Agrarstrukturwandel im Berggebiet. Reckenholz-Tänikon: Agroscope. 217 p.

LEHMANN B, MESSERLI P (2007) The Swiss National Research Programme "Landscapes and habitats of the Alpine Arc". J Alp Res 4: 19-28.

MARINI L, KLIMEK S, BATTISTI A (2011) Mitigating the impacts of the decline of traditional farming on mountain landscapes and biodiversity: a case study in the European Alps. Environ Sci Pol 14: 258-267. 
MILLENNIUM ECOSYSTEM ASSESSMENT (2005) Ecosystems and human well-being: Synthesis. Washington DC: Island Press. $155 \mathrm{p}$.

NEWESELY C, TASSER E, SPADINGER P, CERNUSCA A (2000) Effects of land-use changes on snow gliding processes in alpine ecosystems. Basic Appl Ecol 1: 61-67.

OLSCHEWSKI R, ELSASSER P (2010) Mehr als «Habitat» oder «Dienstleister»? Der Wald als Komponente eines Landschaftssystems. Schweiz Z Forstwes 161: 334-340. doi: 10.3188/ szf. 2010.0334

RENWICK A ET AL (2013) Policy reform and agricultural land abandonment in the EU. Land Use Pol 30: 446-457.

RIGLING A ET AL (2012) Wald und Klimawandel in der inneralpinen Trockenregion Visp. Schweiz Z Forstwes 163: 481-492. doi: 10.3188/szf.2012.0481

SCHUMACHER S, BUGMANN H, MLADENOFF DJ (2004) Improving the formulation of tree growth and succession in a spatially explicit landscape model. Ecol Model 180: 175-194.
SEPPELT R, DORMANN CF, EPPINK FV, LAUTENBACH S, SCHMIDT $S$ (2011) A quantitative review of ecosystem service studies: approaches, shortcomings and the road ahead. J Appl Ecol 48: 630-636.

SOLIVA R, BOLLIGER J, HUNZIKER M (2010) Differences in preferences towards potential future landscapes in the Swiss Alps. Landsc Res 35: 671-696.

STÖCKLIN J, BOSSHARD A, KLAUS G, RUDMANN-MAURER K, FISCHER M (2007) Landnutzung und biologische Vielfalt in den Alpen: Fakten, Perspektiven, Empfehlungen. Zürich: VDF. $191 \mathrm{p}$.

TASSER E, MADER M, TAPPEINER U (2003) Effects of land use in alpine grasslands on the probability of landslides. Basic Appl Ecol 4: 271-280.

ZIMMERMANN W (2012) Waldpolitischer Jahresrückblick 2011. Schweiz Z Forstwes 163: 145-154. doi: 10.3188/szf.2012.0145

\section{Waldausdehnung in zwei Regionen des Schweizer Berggebiets: eine integrative Analyse}

Das Forschungsprojekt «Mountland» untersucht Klima- und Landnutzungsänderungen aus einer ganzheitlichen Perspektive. Das klassische Beispiel für die Interaktion von Land- und Forstwirtschaft ist die Waldausdehnung im Berggebiet, die nur aus dem Verständnis sowohl der sozioökonomischen Entwicklung der Landwirtschaft als auch der naturwissenschaftlichen Grundlagen der Waldentwicklung untersucht werden kann. In diesem Artikel werden die Ergebnisse einer Simulation der Waldausdehnung mit dem Landnutzungsmodell «Aluam» für die Fallbeispielregionen Jura (Waadt) und Visp (Wallis) unter einem Klima- und zwei Politikszenarien (Status quo respektive Liberalisierungen in der Landwirtschaft) präsentiert und diskutiert. Dabei zeigt sich, dass der Umfang und die Art der Veränderungen nicht nur vom gewählten Politikszenario abhängen, sondern auch von der betrachteten Region. Bei der Politikentwicklung ist es daher wichtig, allfällige Schwellenwerte, die Heterogenität des Systems sowie die Wechselbeziehungen zwischen ökonomischen und ökologischen Leistungen zu berücksichtigen. Wenn die weitere Waldzunahme im Berggebiet vermindert werden soll, bedeutet das, dass die verwendeten Strategien auf die lokalen Verhältnisse abgestimmt werden müssen. Dabei werden die raumplanerischen Methoden und Instrumente zentrale Steuerungselemente sein.

\section{L'extension de la forêt dans deux régions des montagnes suisses: une analyse inté- grative}

Le projet de recherche «Mountland» examine les changements du climat et de I'utilisation des terres sous un angle global. L'extension de la forêt en région de montagne est un exemple classique de l'interaction de l'agriculture et de la sylviculture. Cet aspect ne peut être étudié qu'en connaissant à la fois l'évolution socioéconomique de l'agriculture et les bases écologiques de l'évolution de la forêt. Cet article présente et discute les résultats d'une simulation de l'extension de la forêt, réalisée à l'aide du modèle d'utilisation des terres «Aluam» pour les régions du Jura (Vaud) et de Viège (Valais) qui furent soumises à un scénario climatique et à deux scénarios politiques (statu quo et libéralisation dans l'agriculture). Il en résulte que l'ampleur et le genre des changements ne dépendent pas seulement du scénario politique choisi, mais aussi de la région étudiée. II importe donc que les acteurs politiques prennent en compte désormais les éventuelles valeurs seuils ainsi que l'hétérogénéité du système et les interactions entre les prestations économiques et écologiques. Si l'augmentation de la forêt doit être freinée en région de montagne, cela signifie qu'il faudra adapter les stratégies aux conditions locales. Les méthodes et instruments d'aménagement du territoire seront alors des éléments-clés de cette gestion. 